\title{
A Herbal Formulation of Danshen and Gegen Protects Rat Brains from Injuries Induced by Focal and Global Ischaemia
}

\author{
Deng $\mathrm{Y}^{1}$, Lam $\mathrm{FFY}^{2 *}$, Ng ESK ${ }^{2}$, Lau $\mathrm{CBS}^{3,4}$, Koon $\mathrm{JCM}^{3,4}$, Leung $\mathrm{PC}^{3,4}$ and Fung $\mathrm{KP}^{2,3,4}$ \\ ${ }^{1}$ Department of Obstetrics and Gynecology, Faculty of Medicine, The Chinese University of Hong Kong, Shatin, New Territories, \\ Hong Kong SAR, China \\ ${ }^{2} S c h o o l$ of Biomedical Sciences, Faculty of Medicine, The Chinese University of Hong Kong, Shatin, New Territories, Hong Kong \\ SAR, China \\ ${ }^{3}$ Institute of Chinese Medicine, The Chinese University of Hong Kong, Shatin, New Territories, Hong Kong SAR, China \\ ${ }^{4}$ State Key Laboratory of Phytochemistry and Plant Resources in West China, The Chinese University of Hong Kong, Shatin, New \\ Territories, Hong Kong SAR, China
}

*Corresponding author: Lam FFY, School of Biomedical Sciences, The Chinese University of Hong Kong, Lo Kwee Seong Integrated Biomedical Sciences Building, Room 226, Shatin, New Territories, Hong Kong, China, Fax: +852 2603 5123, Tel: +852 3943 6790, E-mail: francislam@cuhk.edu.hk

Citation: Deng Y, Lam FFY, Ng ESK, Lau CBS, Koon JCM, et al. (2018) A Herbal Formulation of Danshen and Gegen Protects Rat Brains from Injuries Induced By Focal and Global Ischaemia. J Adv Plant Sci 1: 104

Article history: Received: 24 May 2018, Accepted: 24 July 2018, Published: 25 July 2018

\begin{abstract}
Background: Ischaemic stroke is a disease that affects many people in developed countries. The occurrence of this disease is also rising globally. Danshen and Gegen are two commonly used Chinese herbal medicines for the treatment of cardiovascular diseases. This study investigates the efficacy of a combined formulation of Danshen and Gegen (DG) in protecting the rat brains from damages induced by global and focal brain ischaemia.

Methods: Global brain ischaemia in the rat was induced by $10 \mathrm{~min}$ bilateral common carotid artery occlusion with hypotension followed by $24 \mathrm{~h}$ reperfusion, and focal brain ischaemia was induced by 90 min middle cerebral artery occlusion (MCAO) followed by $24 \mathrm{~h}$ reperfusion.

Results: Global ischaemia in the rat brain produced elevated levels of nitrite and malondialdehyde, and decreased the activities of the anti-oxidant enzymes superoxide dismutase, catalase, and reduced glutathione. Daily oral administration of DG ( $0.3 \mathrm{~g} / \mathrm{kg}$ and $3 \mathrm{~g} / \mathrm{kg})$ for 7 days prior to the induction of global ischaemia produced dose-dependent suppression on the production of nitrite and malondialdehyde, and restored activities of the anti-oxidant enzymes. Rat brains subjected to focal ischaemia showed increased brain infarct size, infarct weight, and neurological deficit. These detrimental changes were markedly attenuated by the same pretreatment protocol with DG (3 g/ $\mathrm{kg})$.

Conclusion: The present DG formulation was found to possess anti-nitrative and anti-oxidative stress activities that could suppress ischaemia-induced damages in the rat brain. Therefore, the combined use of Danshen and Gegen for treatment of ischaemic stroke is favourable.
\end{abstract}

Keywords: Chinese herbal formula; Danshen; Gegen; Stroke; Global ischaemia; Focal ischaemia

\section{Introduction}

Danshen is the dried root of Salvia miltiorrhiza. It is the first Chinese herbal medicine that received Food and Drug Administration (FDA) approval in the United States. This herb was used for the treatment of cardiovascular diseases in China for many years [1-3]. It is still highly valued and remains to be one of the most widely prescribed single-herbal remedies by modern Practitioners of Traditional Chinese medicine (TCM). Gegen, the dried root of Pueraria lobata, is another Chinese medicinal herb commonly used for the treatment of cardiovascular and other diseases [4,5]. 
Danshen and Gegen are both listed in the Chinese Pharmacopoeia (The Pharmacopoeia of PR China, 2010). These two herbs can be used separately or as a combined formulation for the treatment of coronary heart diseases [6-8]. Recent evidence suggests these two herbs possess anti-oxidative and vasodilator properties, and they can improve arthrogenic pathophysiology by curtailing early atherosclerotic events [9-12]. In addition, a Danshen and Gegen (DG) formulation was shown to improve lipid profile, as well as the vascular structure and function in patients with coronary diseases [13]. We suspect that these cardiovascular benefits of DG can be extrapolated to the cerebrovascular system, but there is limited investigation on the combined use of DG in cerebrovascular diseases.

According to the World Health Organisation, stroke is rank as the second leading cause of death in 2015 [14]. Stroke is a condition characterized by compromised blood flow to the brain, and depending on its etiology, it is classified as ischaemic stroke or haemorhagic stroke. The former is caused by obstruction of blood supply to the brain such as that resulted from atherosclerosis or embolism. On the other hand, haemorhagic stroke is caused by bleeding such as that resulted from the rupture of a cerebral blood vessel. Ischaemic stroke accounts for over $80 \%$ of stroke cases.

Treatment of ischaemic stroke is largely dependent on the use of recombinant tissue plasminogen activator (tPA), which has a high risk of producing haemorrhagic side effects. Moreover, owing to a narrow therapeutic window, tPA is preferred to be used within 3 to 4.5 hours after the onset of ischaemia [15-17]. Recent clinical trials showed that surgical removal of the blood clot together with thrombolysis can achieved a better success rate than thrombolysis therapy alone. However, the procedure of thrombectomy is invasive and it can be applied only to a small population of stroke patients with impaired anterior circulation and occlusion of large artery over $8 \mathrm{~mm}$ in length $[18,19]$. Clearly, current approaches for treatment of stroke patients are less than ideal, and it is imperative to search for better treatment options for this devastating disease.

We have previously shown that both Danshen and Gegen can dilate rat cerebral basilar arteries and their vasodilator effects are additive when used together [20]. This prompts us to speculate that a DG formulation might help patients with obstructive cerebral vascular diseases. Therefore, in this study, we aim to determine whether a DG formulation can provide protective actions in rat models of ischaemic stroke.

\section{Materials and Methods}

\section{Plant materials and chemicals}

Danshen and Gegen, in the form of dried herbs, were purchased from local herbal shops in Sichuan and Guangdong (China), respectively. Water extracts of Danshen and Gegen (DG) were prepared as previously described [13]. Briefly, the raw materials of Danshen and Gegen were washed, cut and dried. Danshen and Gegen were mixed in the ratio of 7:3 and allowed to soak with 10 -fold of water $(\mathrm{v} / \mathrm{w})$ for $1.5 \mathrm{~h}$ followed by extraction at $100^{\circ} \mathrm{C}$ for $1 \mathrm{~h}$. Two subsequent extractions were carried out by adding 8 -fold of water $(\mathrm{v} / \mathrm{w})$ and extracted for 1 and $0.5 \mathrm{~h}$. The extracts were collected and combined and then concentrated at $65{ }^{\circ} \mathrm{C}$ under reduced pressure of $-0.07 \mathrm{MPa}$ to give dry powder with a yield of $10.1 \%$. A specimen voucher of the present Danshen and Gegen (DG) formulation (batch\#: CaoHui27082008-H2O) is kept in the museum of Institute of Chinese Medicine, The Chinese University of Hong Kong. Assay kits for nitrite, malondialdehyde (MDA), catalase (CAT), reduced glutathione (GSH), and superoxide dismutase (SOD) activities were purchased from Nanjing Jiancheng Bioengineering Institute, China. Chloride hydrate and 2, 3, 5-triphenyltetrazolium chloride (TTC) were the product of Sigma, USA.

\section{Animals}

The present studies were performed on male Sprague-Dawley (SD) rats (260-290 g) bred at the Chinese University of Hong Kong. The ARRIVE guidelines for reporting experiments involving animals were strictly followed [21,22]. Experimental protocols were approved by the Animal Experimentation Ethics Committee of the Chinese University of Hong Kong, and licensed by the Department of Health of Hong Kong.

\section{Experimental protocols for global and focal brain ischaemia}

Rats had free access to water and food. They were fed with $0.3 \mathrm{~g} / \mathrm{kg}$ DG (human equivalent dosage), or $3 \mathrm{~g} / \mathrm{kg}$ DG (10 x human equivalent dosage), or vehicle by oral gavage daily for 7 days prior to induction of global or focal brain ischaemia. For the induction of global brain ischaemia, rats were anaesthetized with chloral hydrate (400 $\mathrm{mg} / \mathrm{kg}$, i.p.) and fixed in a stereotactic frame. The parietal bone was thinned by drilling under saline cooling until the blood vessels can be clearly observed. After scanning the basal cerebral blood flow with a laser Doppler perfusion imager (LDI, Moore Instruments), rats were placed on supine position and both femoral arteries were exposed; the left one was cannulated for blood pressure monitoring and the right one was cannulated for blood withdrawal. Both common carotid arteries were also exposed. The induction of global ischaemia was performed by bilateral occlusion of the common carotid arteries using bulldog clips for 10 min combined with withdrawal of blood from the femoral artery to reduce the blood pressure to 40-45 $\mathrm{mmHg}$. During the course of ischaemia, the cerebral blood flow was scanned for a second time. Subsequently, reperfusion was performed by loosening the bulldog clips and infusion of the withdrawn blood, and cerebral blood flow was scanned. The skin was then closed with stitches using waxed silk suture. Temperature was maintained around $37 \pm 0.5^{\circ} \mathrm{C}$ throughout the surgical procedure using a thermostatic heating pad. The animals were allowed to recover, and at $24 \mathrm{~h}$ after reperfusion, they were sacrificed for biochemical assessment as previously described [23]. Briefly, the collected brain 
cortices were homogenated to obtain supernatants, which were then measured by commercially available kits for the levels of $\mathrm{NO}$ and MDA, and the activities of SOD, CAT, and glutathione peroxidase (GSH-Px). Protein level was determined by the BCA method.

The protocols for induction of focal brain ischaemia and assessments of neurobehavioural deficits and cerebral infarction have been described previously [23]. Essentially, rats were prepared in the same manner as described above, but instead of being subjected to global ischaemia, they were subjected to middle cerebral artery occlusion (MCAO) for $90 \mathrm{~min}$. At $24 \mathrm{~h}$ after reperfusion, the animals were examined for neurobehavioural deficits and cerebral infarction.

\section{Statistical Analysis}

Each experiment was performed at least 5 times. All values were calculated as percentage of sham control (normalized to $100 \%$ ), except for neurological deficit, which was scored on a five-point scale. Differences between treatment groups were analysed by oneway ANOVA followed by Dunnett's post hoc test. Results were presented as the mean \pm standard deviation (SD), and significance level was set at $\mathrm{P}<0.05$.

\section{Results}

\section{DG on cerebral blood flow and systemic blood pressure in global ischaemia model}

In the present global brain ischaemia model, challenging the rats with bilateral common carotid artery occlusion combining with hypotension reduced the cerebral blood flow to $20-30 \%$ of basal level (Figure 1A). Blood flow was restored to basal level immediately after reperfusion and restoration of blood pressure. Pretreatment with DG produced no change on this trend (Figure 1A). Global brain ischaemia followed by $24 \mathrm{~h}$ reperfusion produced a small but significant increase in systemic blood pressure in the rats (Figure 1B). Pretreatment with either $0.3 \mathrm{~g} / \mathrm{kg}$ DG or $3 \mathrm{~g} / \mathrm{kg} \mathrm{DG}$ produced a dose-dependent trend in restoring the systemic blood pressure to normal, but the effect did not reach a significant level (Figure 1B).

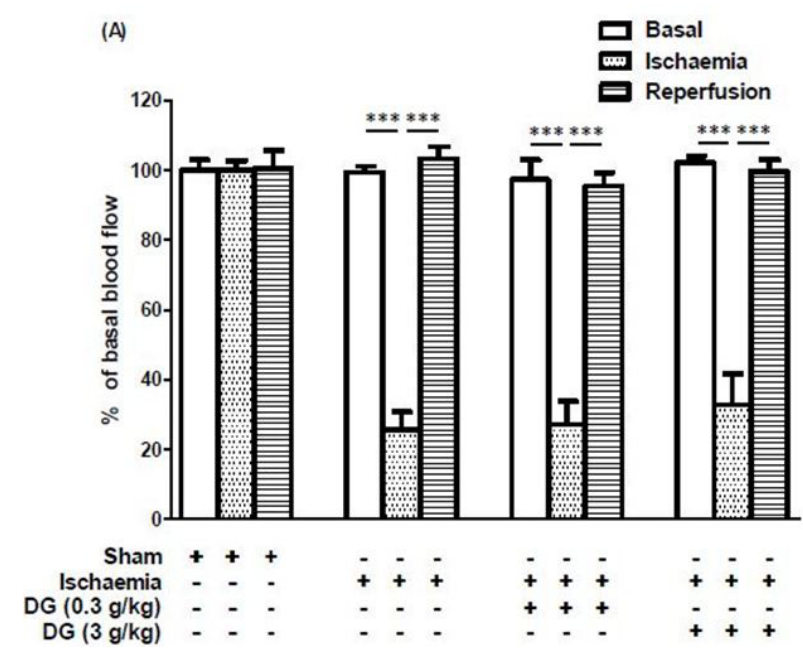

(B)

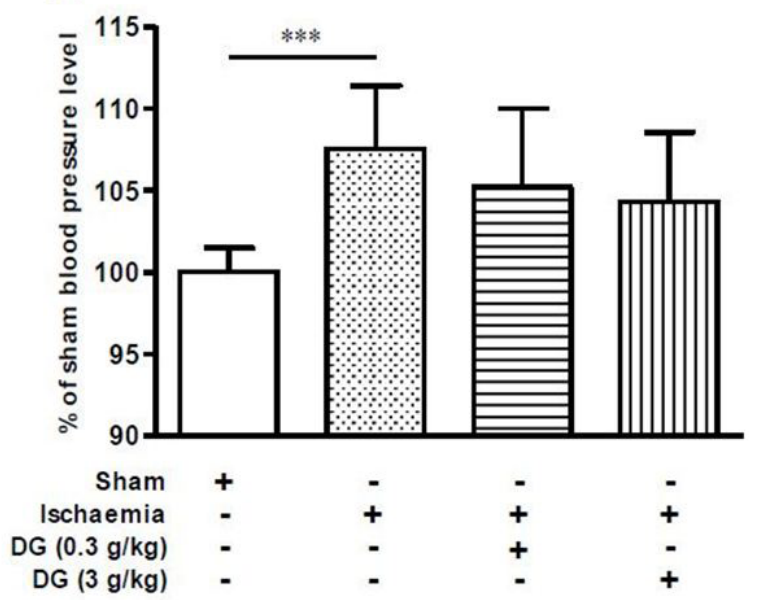

Figure 1: Effects of DG on cerebral blood flow and systemic blood pressure and in rats subjected to global brain ischaemia-reperfusion injury. DG had no significant effect on cerebral blood flow and systemic blood pressure of rats subjected to global brain ischaemia-reperfusion. One-way ANOVA, $* * * P<0.001$. The data are expressed as the mean $\pm S D, n=6-7$ 
DG on lipid peroxidation, SOD and CAT activity, and GSH level in global ischaemia model

Rat brains subjected to global ischaemia showed substantial increases in MDA and nitrite levels, and these increases were abolished by treatment with $3 \mathrm{~g} / \mathrm{kg} \mathrm{DG}$ (Figure 2). On the other hand, the levels of SOD, CAT, and reduced GSH were decreased in the ischaemic brain, and treatment with $3 \mathrm{~g} / \mathrm{kg}$ DG restored the activities of these three anti-oxidant enzymes (Figure 3).

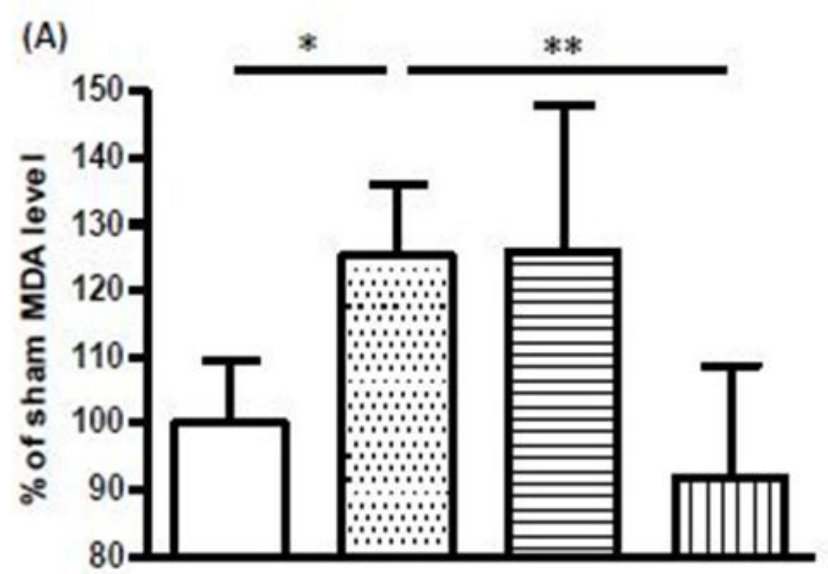

(B)
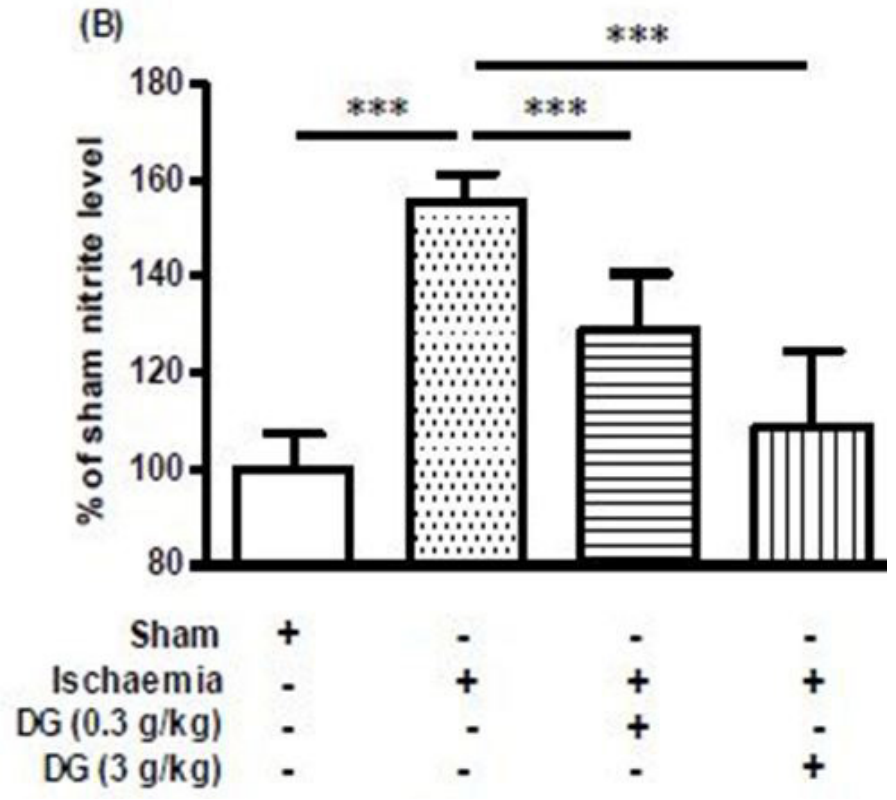

Figure 2: Effects of DG on malondialdehyde (MDA) and nitrite levels in rat brains subjected to global ischaemia-reperfusion injury. DG inhibited the increase in MDA (A) and nitrite levels (B) in the ischaemic brains. One-way ANOVA, $* P<0.05, * * P<0.01, * * * P<0.001$. The data are expressed as the mean $\pm \mathrm{SD}, \mathrm{n}=5-7$

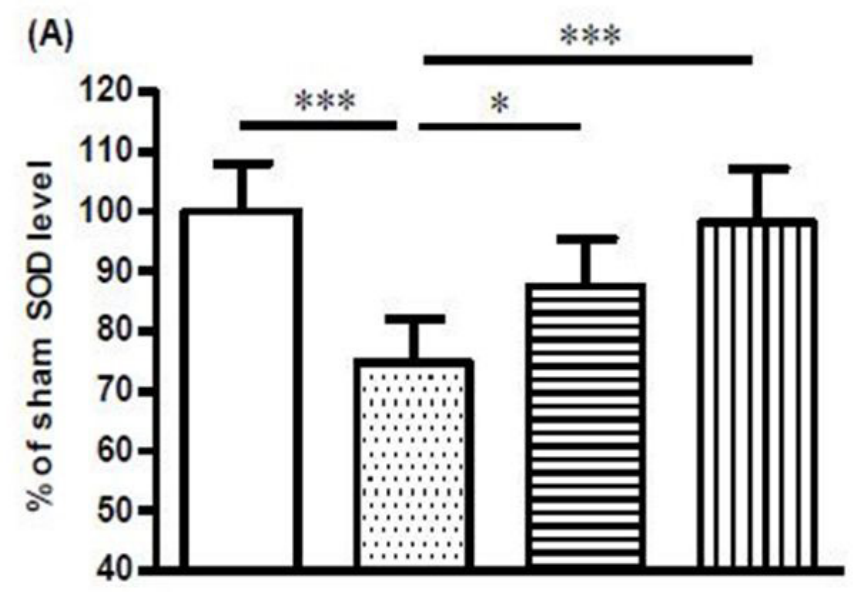



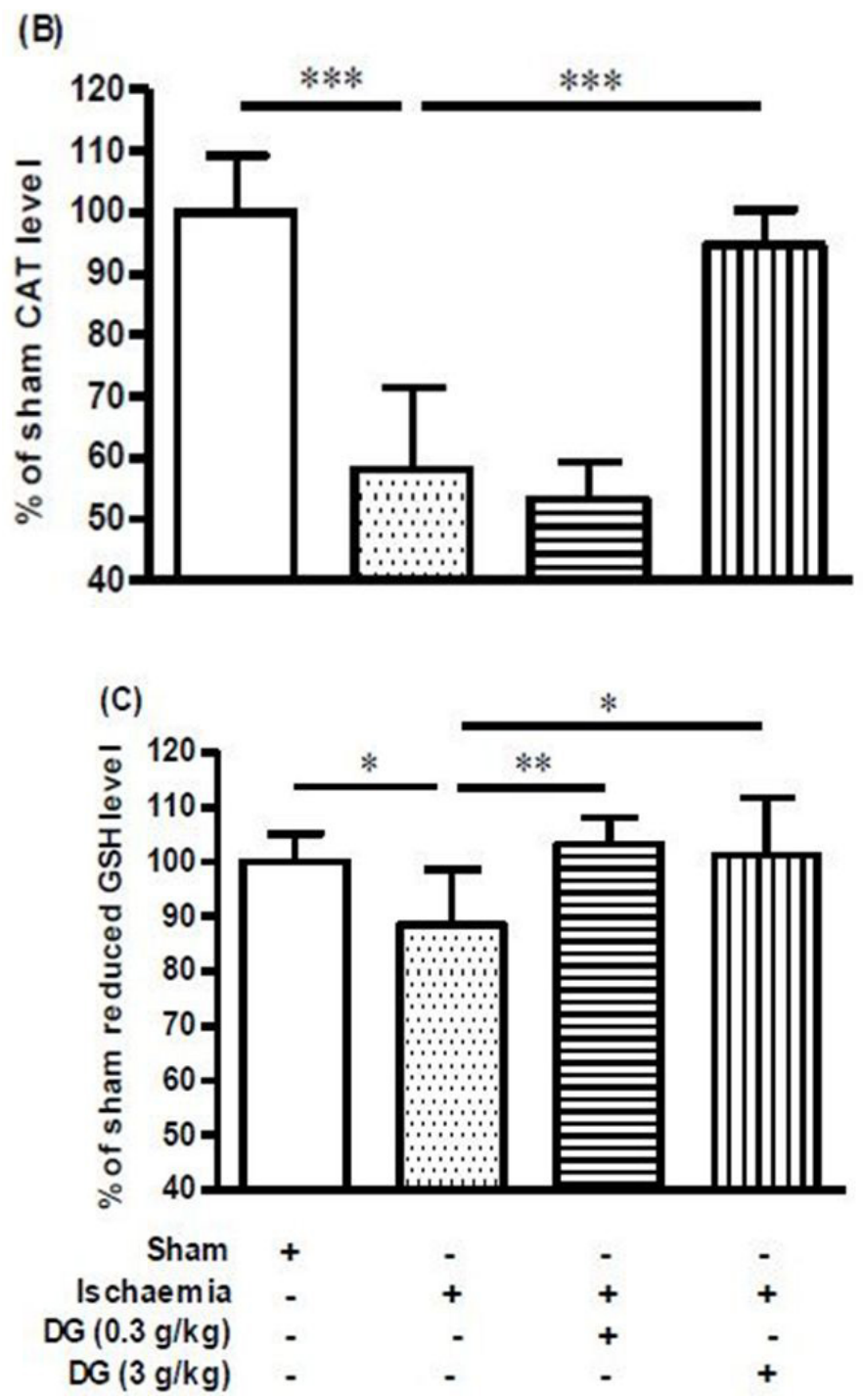

Figure 3: Effects of DG on anti-oxidant enzymes in rat brains subjected to global ischaemia-reperfusion injury. DG restored superoxide dismutase (SOD) (A), catalase $(\mathrm{CAT})(\mathrm{B})$, and reduced glutathione $(\mathrm{GSH})(\mathrm{C})$ levels in the ischaemic brains. One-way ANOVA, $* P<0.05, * * P<0.01, * * * P<0.001$. The data are expressed as the mean $\pm \mathrm{SD}, \mathrm{n}=6-7$

DG on infarct size, infarct weight, and neurological deficit in focal ischaemia model

The ipsilateral side of rat brains subjected to focal brain ischaemia showed marked infarct area and infarct weight (Figure 4A and 4B). These animals also had pronounced neurological deficit (Figure 4C). Treatment with $3 \mathrm{~g} / \mathrm{kg}$ DG reduced the infarct weight from $16.06 \pm 3.59 \%$ to $10.53 \pm 3.528 \%$ (i.e. $34 \%$ reduction, Figure $4 \mathrm{~B}$ ), and reduced the neurological score from $2.40 \pm 0.55$ to 1.67 \pm 0.52 (i.e. $30 \%$ reduction, Figure $4 B$ ).

(A)

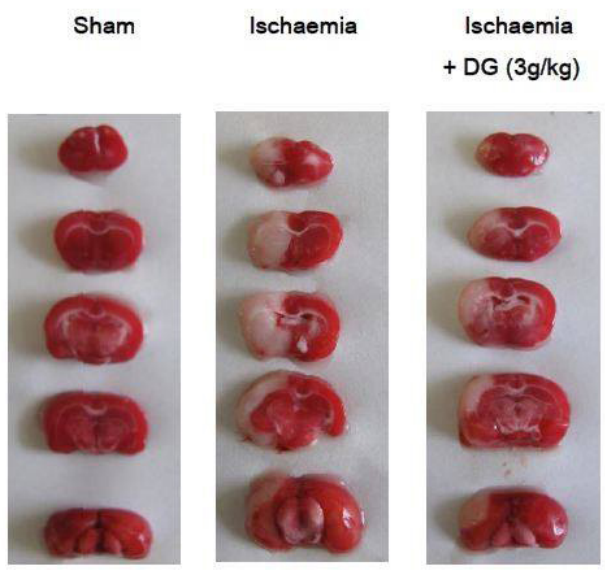



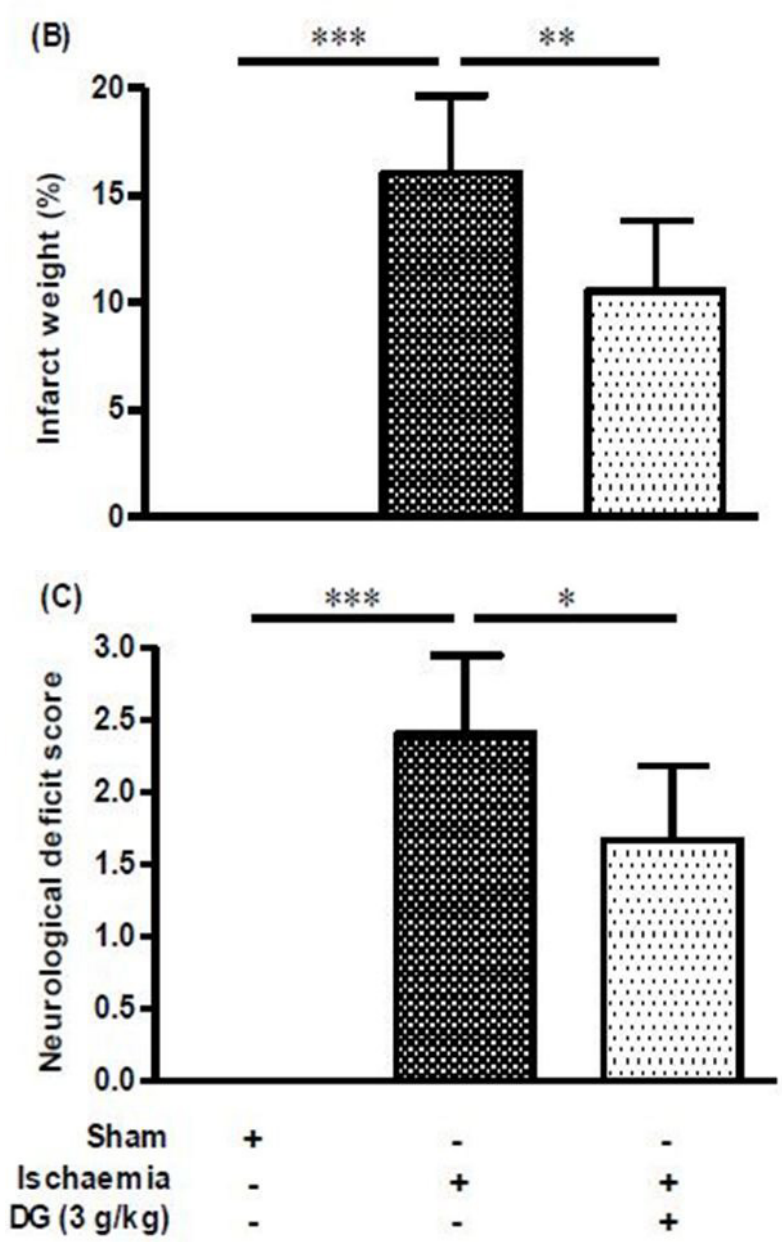

Figure 4: Effects of DG on indexes of brain injuries induced by focal (MCAO)-reperfusion injury. Representative photos of TTC staining of brain slices show that DG reduced the cerebral infarct size (A). DG also significantly reduced infarct weight (B) and neurological deficit (C) in the ischaemic brains. One-way ANOVA, $* P<0.05, * * P<0.01$, $* * * P<0.001$. The data are expressed as the mean \pm SD, $\mathrm{n}=5-6$

\section{Discussion}

Practitioners of TCM often prescribe Danshen and Gegen as a combined therapy for the treatment of coronary heart diseases [6-8]. Modern scientific studies have confirmed that many of the ingredients of these two herbs have protective actions on the cardiovascular system [3,11,24-27]. Information on the combined use of Danshen and Gegen in the treatment of cerebral vascular diseases is limited, but we have recently shown that both Danshen and Gegen can dilate rat cerebral basilar arteries and their vasodilator effects were additive when used in the form of a DG decoction [20]. In the present study, we confirmed that this DG decoction can protect rat brains from injuries induced by ischaemic stroke.

It has been reported that DG can lower systolic blood pressure of spontaneously hypertensive rats (SHR) when given orally at 0.3 $\mathrm{g} / \mathrm{kg}$ [28]. In the present study, global brain ischaemia followed by $24 \mathrm{~h}$ reperfusion produced a small but significant increase in systemic blood pressure in the rats. However, the systemic effect of DG in this stroke model is negligible. Blood flow in different regions of the brain can be affected differently by brain ischaemia. A period of 5 to 15 minutes ischaemia in the rat brain was shown to reduce blood flow in the cerebral cortex by $<5 \%$, whereas, blood flow in the cingulate cortex, hippocampus, and caudoputamen was reduced to $<15 \%$, and blood flow in the globus pallidus, thalamus, and midbrain were affected less and showed more variable changes [29]. In the present global brain ischaemia model, cerebral blood flow was reduced to 20-30\% of basal level, and it can be reverted to basal level after reperfusion and restoration of blood pressure, both in the absence or presence of DG. It is possible that cerebral blood vessels were already maximally dilated under our experimental conditions, and therefore they cannot be dilated further even with drug treatment. Moreover, cerebral autoregulation can help to stablize cerebral blood flow when there are changes in peripheral blood pressure. These factors might account for the lack of effect of DG on basal and reperfusion blood flow in the brain, although DG was shown to have negligible systemic effect in the present stroke model. The complexity of cerebral autoregulation in response to changes in peripheral blood pressure has brought controversial views on the use of vasodilator agents in treatment of stroke patients. While some consider them to be harmful, others have shown that they could improve blood flow in ischaemic brain regions and have neuroprotective effects in experimental stroke models and in stroke patients [30,31]. Thus, more studies are required to ascertain the usefulness of vasodilators in the treatment of stroke patients. 
Transient global ischaemia can be a result of cardiac arrest or surgery. The reperfusion process following transient ischaemia provides oxygen that acts as a substrate for the production of reactive oxygen species (ROS) such as hydrogen peroxide $\left(\mathrm{H}_{2} \mathrm{O}_{2}\right)$, hydroxyl radical $(-\mathrm{OH})$, and superoxide anion radical $\left(\bullet \mathrm{O}_{2}-\right)$. Excessive production of $\mathrm{ROS}$ can have detrimental effect on cell membranes through degradation of polyunsaturated fatty acids (PUFAs) and releasing MDA in the process. Hence, the measurement of MDA levels can be used to assess the extent of membrane damage. Nitrite and nitrate are the major oxidative metabolites of NO. Nitrite represents an attractive estimate of regional NO formation, whereas, nitrate is useful in estimating overall nitrogen/NO turnover [32]. In the present studies, MDA and nitrite levels were substantially increased in rat brains subjected to global ischaemia. This confirmed the occurrence of oxidative and nitrative stress in the ischaemic brain.

Under normal physiological condition, there is a homeostatic balance between oxidative radicals and antioxidant enzymes. This ensures that the detrimental effects of ROS cannot be manifested. However, oxidative stress can affect not only the levels of ROS but also the levels of antioxidant enzymes [33]. Accordingly, apart from an increase in MDA and nitrite levels, the ischaemic brains also showed reduced levels of the antioxidant enzymes, SOD, CAT, and reduced GSH. More importantly, treatment with DG suppressed the increase in MDA and nitrite levels, while the levels of the three anti-oxidant enzymes were restored. These findings confirmed DG to be an effective agent in suppressing nitrative and oxidative stress in the ischaemic brain. In line with the present findings, a previous study using a 4-vessel occlusion rat global ischaemia model showed that pre-treatment with Danshen suppressed the ischaemia-induced increase in cerebral NO and MDA levels, and significantly restored the decreased SOD activity $[34,35]$. In another ischaemia/reperfusion rat model, pretreatment with Danshen reduced brain oedema and MDA concentration, and increased levels of catalase, SOD, GSH and ATP in the cerebral cortex and hippocampus region [36].

Nowadays, the incidence of stroke has been reduced by preventive measures, such as controlling hypertension, hypercholesterolaemia, substance abuse and smoking as well as the use of anticoagulation drugs in some high-risk patients [37]. Treatments aiming at dissolving thrombus and restoring blood flow, and therapies which can prevent the ischaemic inflammatory response, block excitatory neurotransmission or scavenge free radicals have all shown promising therapeutic potential in animal stroke models [38]. Recombinant tissue plasminogen activator (tPA), a clot-dissolving drug, has been demonstrated to be an effective emergency treatment for ischaemic stroke. However, owing to a narrow therapeutic window, thrombolytic therapy with recombinant $\mathrm{t}-\mathrm{PA}$ can only be used within 3 to 4 .5h of the onset of ischaemia [15-17]. t-PA therapy should be used with caution because it carries a high risk of producing haemorrhagic side effects, and it is therefore, unsuitable for patients with haemorrhagic stroke. These requirements limit the use of t-PA therapy and only 1-2\% of all stroke patients' benefit from it [39]. Overall, no currently approved treatment consistently reduces stroke size or neurological disability in human beings.

Stroke caused by obstruction of the middle cerebral artery (MCA) carries a poor prognosis because it reduces oxygen-rich blood supply to the brainstem. The resulted hypoxia and ischaemia could lead to paralysis of all extremities, heavy disturbances in sensation, difficulty in swallowing and difficulty in respiration. Performing middle cerebral artery occlusion (MCAO) in rats can mimic such stroke condition in humans. The simplicity of this procedure makes it the most popular rat model for the study of ischaemic stroke. The MCAO model typically induces infarcts in the lateral caudatoputamen and frontoparietal cortex, and because it produces a substantial amount of penumbra (salvageable tissue) in the first 60-90 minutes after onset, it is suitable for neuroprotection drug experiments [40].

In the present study, we have demonstrated that the DG formulation has neuroprotective activity on cerebral ischaemia in the rat MCAO model. Infarction is an obvious pathophysiological change that can be observed in cerebral ischaemia. Analysis of the infarct area by TTC staining has been commonly employed to determine the efficacy of cerebroprotective compounds in pre-clinical trials. TTC is a sensitive histochemical indicator of mitochondrial respiratory enzyme function, which is a colourless chemical that is reduced by mitochondrial enzymes into a compound that stains intact brain regions dark red, whereas, infarcted regions remain white. Therefore, brain lesion identified by TTC staining indicates that mitochondrial function and oxidative respiratory enzyme systems in the tissues were irreversibly impaired. Using the TTC staining method in the rat MCAO model, DG was shown to produce significant reductions in infarct size and weight, and improved neurological deficit. In agreement with the present findings, Danshen on its own has also been shown to reduce cerebral infarction volume and neurological deficit score, and its major constituents, tanshinone IIA, exhibited neuroprotective effects against focal cerebral ischaemic-reperfusion injuries in rats [41-43]. Salvianolic acid B, another constituent of Danshen, was also reported to stimulate neurogenesis both in sub-granular zone and sub-ventricular zone, alleviate neural cells loss, and improve motor function recovery after brain ischaemia-reperfusion in rats [44]. Moreover, puerarin, a major constitute of Gegen, was shown to be a potent neuroprotective agent on MCAO-induced focal cerebral ischaemia, and it significantly depressed ischaemia-induced efflux of amino acids and excitotoxicity in the striatum $[45,46]$. These findings irrevocably demonstrate that many of the constituents in DG have protective actions on ischaemia-induced brain injuries, and thus lends support to the use of DG in the treatment of ischaemic cerebrovascular diseases.

\section{Conclusion}

The present study has demonstrated that a DG formulation has protective effects on rat brains subjected to global and focal brain ischaemia. The beneficial effects of DG are manifested as reductions in nitrative and oxidative stress in the globally ischaemic 
brain, and curtailment of neurological deficits and infarct volume in the focally ischaemic brain. Therefore, this DG formulation represents a natural product that could be potentially useful in the treatment of ischaemic stroke.

\section{Acknowledgement}

The work described in this paper was supported by a grant from University Grant Committee Area of Excellence project "Chinese Medicine Research and Further Development” of the Hong Kong Special Administrative Region, China (Project No. AoE/B-10/01).

\section{References}

1. Cheng TO (2006) Danshen: a versatile Chinese herbal drug for the treatment of coronary heart disease. Int J Cardiol 113: 437-38.

2. Cui SK, Li GH (2003) Clinical effect of Fufang Danshen Dripping Pill on hyperlipidemia. Xiandai Zhongxiji Jiehe Zazhi 11: 1029-30.

3. Ji XY, Tan BK, Zhu YZ (2000) Salvia miltiorrhiza and ischemic diseases. Acta Pharmacol Sin 21: 1089-94.

4. Song J, Han Q, Qiao C, Yip Y, Xu H (2007) Simultaneous determination of multiple marker constituents in concentrated Gegen Tang granule by high performance liquid chromatography. Chin Med 2: 7.

5. Wong KH, Li GQ, Li KM, Razmovski-Naumouski V, Chan K (2011) Kudzu root: traditional uses and potential medicinal benefits in diabetes and cardiovascular diseases. J Ethnopharmacol 134: 584-607.

6. Chen YM (2002) A clinical practice of Jiang-Zhi-Yin on therapeutic effects of lipid disorder. Zhonghua Yixue Zazhi 2: 1020-1.

7. Ren JS, Luo XH (2006) A review on the combination uses of Gegen and Danshen in traditional Chinese medicine. Shanxi Zhongyi Xueyuan Xuebao 7: 51-2.

8. Zhang WX, Ma DL, Zhang Y, Gao Y (1991) The studies on the stability of a Chinese medicine Guan-Xin tablet. Zhongguo Zhongyao Zazhi 16: 284-5.

9. Lam HM, Yam WS, Lau KM, Leung L.K., Koon CM, et al. (2005) Antioxidative and vasodilative effects of danshen and gegen. J Mol Cell Cardiol 38: 840.

10. Chan YL, Woo KS, Leung PC, Fung KP (2006) Traditional Chinese medicine Danshen-Gegen combination formula improves atherogenic pathophysiology: an in-vitro and ex-vivo study. J H K Coll Cardiol 14: 28.

11. Cheung DWS, Koon CM, Ng CF, Leung PC, Fung KP, et al. (2012) The roots of Salvia miltiorrhiza (Danshen) and Pueraria lobata (Gegen) inhibit atherogenic events: A study of the combination effects of the 2 herb formula. J Ethnopharmacol 143: 859-66.

12. Sieveking DP, Woo KS, Fung KP, Lundman P, Nakhla S, et al. (2005) Chinese herbs Danshen and Gegen modulate key early atherogenic events in vitro. Int J Cardiol 105: 40-5.

13. Tam WY, Chook P, Qiao M, Chan LT, Chan TY, et al. (2009) The efficacy and tolerability of adjunctive alternative herbal medicine (Salvia miltiorrhiza and Pueraria lobata) on vascular function and structure in coronary patients. J Altern Complem Med 15: 415-21.

14. Global Health Estimates. Geneva: World Health Organization (2018).

15. Bright R, Mochly-Rosen D (2005) The role of protein kinase C in cerebral ischemic and reperfusion injury. Stroke 36: 2781-90.

16. Davis SM, Donnan GA (2009) 4.5 hours: the new time window for tissue plasminogen activator in stroke. Stroke 40: 2266-7.

17. Ginsberg MD (2008) Neuroprotection for ischemic stroke: past, present and future. Neuropharmacol 55: 363-89.

18. Meyne JK, Zimmermann PR, Rohr A, Riedel C, Hansen HC, et al. (2015) Thrombectomy vs. Systemic Thrombolysis in Acute Embolic Stroke with High Clot Burden: A Retrospective Analysis. Rofo 187: 555-60.

19. Sardar P, Chatterjee S, Giri J, Kundu A, Tandar A, et al. (2015) Endovascular therapy for acute ischaemic stroke: a systematic review and meta-analysis of randomized trials. Eur Heart J 36: 2373-80.

20. Deng Y, Ng ESK, Kwan YW, Lau CBS, Cheung DWS, et al. (2014) Cerebral vasodilator properties of Danshen and Gegen: A study of their combined efficacy and mechanisms of actions. Phytomedicine 21: 391-9.

21. Kilkenny C, Browne W, Cuthill IC, Emerson M, Altman DG (2010) Animal research: reporting in vivo experiments; the ARRIVE guidelines. B J Pharmacol 160: 1577-9.

22. McGrath J, Drummod G, McLachlan E, Kilkenny C, Wainwright C (2010) Guidelines for reporting experiments involving animals: the ARRIVE guidelines. Br J Pharmacol 160: 1573-6.

23. Zhen X, Ng ESK, Lam FFY (2016) Suppression of ischemia-induced injuries in rat brain by protease-activated receptor-1 (PAR-1) activating peptide. Eur J Pharmacol 786: 36-46.

24. Adams JD, Wang R, Yang J, Lien EJ (2006) Preclinical and clinical examinations of Salvia miltiorrhiza and its tanshinones in ischemic conditions. Chin Med 1: 3.

25. Lam FF, Yeung JH, Chan KM, Or PM (2007) Relaxant effects of danshen aqueous extract and its constituent danshensu on rat coronary artery are mediated by inhibition of calcium channels. Vas Pharmacol 46: 271-7.

26. Lam FFY, Yeung JHK, Chan KM, Or PMY (2008) Mechanisms of the dilator action of cryptotanshinone on rat coronary artery. Eur J Pharmacol 578: 253-60.

27. Lam FFY, Yeung JHK, Chan KM, Or PMY (2008) Dihydrotanshinone, a lipophilic component of Salvia miltiorrhiiza (danshen), relaxes rat coronary artery by inhibition of calcium channels. J Ethnopharmacol 119: 318-21.

28. Ng CF, Koon CM, Cheung DW, Lam MY, Leung PC, et al. (2011) The anti-hypertensive effect of Danshen (Salvia miltiorrhiza) and Gegen (Pueraria lobata) formula in rats and its underlying mechanisms of vasorelaxation. J Ethnopharmacol 137: 1366-72.

29. Ginsberg MD, Busto R, Harik SI (1985) Regional blood-brain barrier permeability to water and cerebral blood flow during status epilepticus: insensitivity to norepinephrine depletion. Brain Res 337: 59-71.

30. Maniskas ME, Roberts JM, Aron I, Fraser JF, Bix GJ (2016) Stroke neuroprotection revisited: Intra-arterial verapamil is profoundly neuroprotective in experimental acute ischemic stroke. J Cereb Blood Flow Metab 36: 721-30.

31. Mchenry LC (1972) Cerebral Vasodilator Therapy in Stroke. Stroke 3: 686-91.

32. Kelm M (1999) Nitric oxide metabolism and breakdown. Biochim Biophys Acta 1411: 273-89.

33. Dong XX, Wang Y, Qin ZH (2009) Molecular mechanisms of excitotoxicity and their relevance to pathogenesis of neurodegenerative diseases. Acta Pharmacol Sin 30: 379-87.

34. Kuang P, Tao Y, Tian Y (1996) Radix Salviae miltiorrhizae treatment results in decreased lipid peroxidation in reperfusion injury. J Tradit Chin Med 16: 138-42. 
35. Kuang P, Tao Y, Tian Y (1996) Effect of radix Salviae miltiorrhizae on nitric oxide in cerebral ischemic-reperfusion injury. J Tradit Chin Med 16: 224-7.

36. Mo Z, Zheng Y, Pan Z, Liang R (1998) Protective effect and mechanism of salvia miltiorrhiza on cerebral ischemia reperfusion injury in rats. Pharmacol Clin Chin Mat Med 14: 24-6.

37. Fisher M (1995) Potentially effective therapies for acute ischemic stroke. Eur Neurol 35: 3-7.

38. Meldrum BS (1995) Cytoprotective therapies in stroke. Curr Opin Neurol 8: 15-23.

39. Beresford IJ, Parsons AA, Hunter AJ (2003) Treatments for stroke. Expert Opin Emerging Drugs 8: 103-22.

40. Meng X, Fisher M, Shen Q, Sotak CH, Duong TQ (2004) Characterizing the diffusion/perfusion mismatch in experimental focal cerebral ischemia. Ann Neurol 55: 207-12.

41. Lao CJ, Lin JG, Kuo JS, Chiang SY, Chen SC, et al. (2003) Effects of Salvia Miltiorrhiza Bunge on cerebral infarct in ischemia-reperfusion injured rats. Am J Chin Med 31: 191-200.

42. Chen Y, Wu X, Yu S, Fauzee NJ, Wu J, et al. (2012) Neuroprotective capabilities of Tanshinone IIA against cerebral ischemia/reperfusion injury via anti-apoptotic pathway in rats. Biol Pharm Bull 35: 164-70.

43. Yang L, Zhang B, Yin L, Cai B, Shan H, et al. (2011) Tanshinone IIA prevented brain iron dyshomeostasis in cerebral ischemic rats. Cell Physiol Biochem 27: 23-30.

44. Zhong J, Tang MK, Zhang Y, Xu QP, Zhang JT (2007) Effect of salvianolic acid B on neural cells damage and neurogenesis after brain ischemia-reperfusion in rats. Yao Xue Xue Bao 42: 716-21.

45. Chang Y, Hsieh CY, Peng ZA, Yen TL, Hsiao G, et al. (2009) Neuroprotective mechanisms of puerarin in middle cerebral artery occlusion-induced brain infarction in rats. J Biomed Sci 16: 9.

46. Xu XH, Zheng XX, Zhou Q, Li H (2007) Inhibition of excitatory amino acid efflux contributes to protective effects of puerarin against cerebral ischemia in rats. Biomed Environ Sci 20: 336-42. 